\title{
EXISTENCE OF OPTIMAL CONTROL FOR NONLINEAR SYSTEMS WITH QUADRATIC PERFORMANCE
}

\author{
K. BALACHANDRAN ${ }^{1}$ AND D. SOMASUNDARAM ${ }^{1}$
}

(Received 3 February 1986; revised 21 May 1986)

\begin{abstract}
We prove the existence of optimal control for nonlinear systems having implicit derivative with quadratic performance criteria.
\end{abstract}

\section{Introduction}

The theory of optimal control of linear systems with quadratic performance criteria is well developed, where the results are most complete and closest to use in practical design. The optimal regulator problem for nonlinear systems has received little attention. For example, Lukes [5] discussed the problem via Lyapunov-like theory. Yamamoto [7] approached the problem by means of the contraction mapping principle and proposed a new control scheme to obtain a quasi-feedback optimal control for nonlinear systems with quadratic performance. Colonius and Hinrichsen [2] investigated the optimal control of functional differential systems via the Dubovitskii-Milyutin Method.

Dacka [4] has introduced a new method of analysis to study the controllability of nonlinear systems with implicit derivative, based on the measure of noncompactness of a set and the Darbo fixed point theorem. The aim of this paper is to prove the existence theorem for optimal control of nonlinear systems having implicit derivative with quadratic performance criteria, by suitably adopting the technique of Dacka [4]. We shall prove the theorem for a larger class of dynamical systems as compared with Yamamoto [7].

\footnotetext{
${ }^{1}$ Department of Mathematics, Madras University PG Centre, Salem 636 011, Tamil Nadu, India. Copyright Australian Mathematical Society 1987, Serial-fee code 0334-2700/87
} 


\section{Mathematical preliminaries}

Let $(X,\|\cdot\|)$ be a Banach space and $E$ be a bounded set of $X$. In this paper the following definition of the measure of noncompactness of a set $E$ is used [6]

$$
\begin{array}{r}
\mu(E)=\inf \{r>0 ; E \text { can be covered by a finite number of } \\
\text { balls whose radii are smaller than } r\} .
\end{array}
$$

Its importance follows from Darbo's theorem which states that, "If $S$ is a nonempty bounded closed convex subset of $X$ and $P: S \rightarrow S$ is a continuous mapping such that for any set $E \subset S$ we have

$$
\mu(P E) \leqslant k \mu(E),
$$

where $k$ is a constant, $0 \leqslant k<1$, then $P$ has a fixed point".

For the space of continuous functions $C_{n}\left[t_{0}, t_{1}\right]$ with norm

$$
\|x\|=\max \left\{\left|x_{i}(t)\right|: i=1, \ldots, n, t \in\left[t_{0}, t_{1}\right]\right\},
$$

the measure of noncompactness of a set $E$ is given by

$$
\mu(E)=\frac{1}{2} w_{0}(E)=\frac{1}{2} \lim _{h \rightarrow 0^{+}} w(E, h),
$$

where $w(E, h)$ is the common modulus of continuity of the functions which belong to the set $E$, that is,

$$
w(E, h)=\sup _{x \in E}[\sup |x(t)-x(s)|:|t-s| \leqslant h],
$$

and for the space of continuously differentiable functions $C_{n}^{1}\left[t_{0}, t_{1}\right]$ with norm

$$
\|x\|_{C_{n}^{1}}=\|D x\|_{C_{n}}+\|x\|_{C_{n}}
$$

we have

$$
\mu(E)=\frac{1}{2} w_{0}(D E)
$$

where

$$
D E=\{\dot{x}: x \in E\} .
$$

Let us form the Cartesian product

$$
C_{n+m}^{1}\left[t_{0}, t_{1}\right]=C_{n}^{1}\left[t_{0}, t_{1}\right] \times C_{m}\left[t_{0}, t_{1}\right]
$$

with the following norm:

$$
\|(x, y)\|_{C_{n+m}^{1}}=\max \left[\|x\|_{C_{n}^{1}},\|y\|_{C_{m}}\right] .
$$


The measure of noncompactness of any set $E$ bounded in $C_{n+m}^{1}\left[t_{0}, t_{1}\right]$ is given by the relation

$$
\mu(E)=\max \left[\mu\left(E_{1}\right), \mu\left(E_{2}\right)\right],
$$

where $E_{1}$ and $E_{2}$ are the natural projections of the set $E$ on the spaces $C_{n}^{1}$ and $C_{m}$.

\section{Basic assumptions}

Consider the nonlinear system

$$
\dot{x}(t)=A(t) x(t)+B(t) u(t)+f(t, x(t), \dot{x}(t))
$$

with initial condition $x\left(t_{0}\right)=x_{0}$ and quadratic performance

$$
J=\frac{1}{2} x^{\prime}\left(t_{1}\right) F x\left(t_{1}\right)+\frac{1}{2} \int_{t_{0}}^{t_{1}}\left[x^{\prime} Q(s) x+u^{\prime} R(s) u\right] d s,
$$

where $x$ is an $n$-vector, $u$ is an $m$-vector, $A(t), B(t)$ and $f(t, x(t), \dot{x}(t))$ are dimensionally appropriate continuous functions. Further $F, Q(s), R(s)$ are respectively constant, positive semidefinite and positive definite dimensionally appropriate matrices with continuous entries. The prime indicates the matrix transpose. Assume that

$$
\|A(t)\| \leqslant M, \quad\|B(t)\| \leqslant N \quad \text { for } t \in\left[t_{0}, t_{1}\right]
$$

with fixed times $t_{0}$ and $t_{1}$,

$$
|f(t, x, y)| \leqslant L \quad \text { for } t \in\left[t_{0}, t_{1}\right] \text { and } x, y \in R^{n} \text {, }
$$

and for every $y, \bar{y} \in R^{n}$ and $x \in R^{n}, t \in\left[t_{0}, t_{1}\right]$,

$$
|f(t, x, y)-f(t, x, \bar{y})| \leqslant k_{1}|y-\bar{y}|,
$$

where $M, N, L$ and $k_{1}$ are positive constants such that $0 \leqslant k_{1}<\frac{1}{2}$. Define the norm of a continuous $n \times m$ matrix valued function $D(t)$ by

$$
\|D(t)\|=\max _{i} \sum_{j=1}^{m}\left|d_{i j}(t)\right|, \text { where } d_{i j} \text { are the elements of } D .
$$

In general, the optimal regulator problem of (1) and (2) can not be solved analytically, hence for each fixed $z \in C_{n}^{1}\left[t_{0}, t_{1}\right]$, consider the following system

$$
\dot{x}(t)=A(t) x(t)+B(t) u(t)+f(t, \dot{z}(t), z(t)) .
$$

Since this system is linear, we can analytically solve the regulator problem for (2) and (6) and obtain the following results [1]:

$$
\begin{aligned}
u(t, z) & =-K^{*}(t) x(t)-g^{*}(t, z, \dot{z}) \\
& =-R^{-1}(t) B^{\prime}(t) K(t) x(t)^{\prime}-R^{-1}(t) B^{\prime}(t) g(t, z, \dot{z}),
\end{aligned}
$$


where

$$
\begin{aligned}
\dot{K}(t) & =-K(t) A(t)-A^{\prime}(t) K(t) B(t) R^{-1}(t) B^{\prime}(t) K(t)-Q(t), \\
K\left(t_{1}\right) & =F, \\
\dot{g}(t, z, \dot{z}) & =-\left[A^{\prime}(t)-K(t) B(t) R^{-1}(t) B^{\prime}(t)\right] g(t, z, \dot{z})-K(t) f(t, z, \dot{z}), \\
g\left(t_{1}, z, \dot{z}\right) & =0 .
\end{aligned}
$$

For this linear regulator problem, if there exists a solution $x(t)$ which agrees with a predetermined function $z(t)$, then this function is also recognized as a solution for the original problem of (1) and (2).

\section{Existence theorem}

THEOREM. If the nonlinear system (1) with quadratic performance (2) satisfies the conditions (3) to (5), then the optimal control exists and is given by

$$
\begin{aligned}
u(t, x) & =-K^{*}(t) x(t)-g^{*}(t, x, \dot{x}) \\
& =-R^{-1}(t) B^{\prime}(t) K(t) x(t)-R^{-1}(t) B^{\prime}(t) g(t, x, \dot{x}),
\end{aligned}
$$

where

$$
\begin{aligned}
\dot{K}(t) & =-K(t) A(t)-A^{\prime}(t) K(t)+K(t) B(t) R^{-1}(t) B^{\prime}(t) K(t)-Q(t), \\
K\left(t_{1}\right) & =F,
\end{aligned}
$$

and

$$
\begin{aligned}
\dot{g}(t, x, \dot{x}) & =-\left[A^{\prime}(t)-K(t) B(t) R^{-1}(t) B^{\prime}(t)\right] g(t, x, \dot{x})-K(t) f(t, x, \dot{x}), \\
g\left(t_{1}, x, \dot{x}\right) & =0 .
\end{aligned}
$$

Proof. The solution of (6) with initial condition $x\left(t_{0}\right)=x_{0}$ is given by

$$
x(t)=F\left(t, t_{0}\right) x_{0}+\int_{t_{0}}^{t} F(t, s) B(s) u(s) d s+\int_{t_{0}}^{t} F(t, s) f(s, z, \dot{z}) d s,
$$

where $F\left(t, t_{0}\right)$ is the fundamental matrix solution for the homogeneous linear equation of (6). Substituting (7) into (13), we obtain

$$
\begin{aligned}
x(t)= & F\left(t, t_{0}\right) x_{0}-\int_{t_{0}}^{t} F(t, s) B(s) K^{*}(s) x(s) d s \\
& -\int_{t_{0}}^{t} F(t, s) B(s) g^{*}(s, z, \dot{z}) d s+\int_{t_{0}}^{t} F(t, s) f(s, z, \dot{z}) d s .
\end{aligned}
$$


with

$$
\begin{gathered}
L_{1}=\sup _{s \in\left[t_{0}, t_{1}\right]}\left\|K^{*}(s)\right\|, \\
L_{2}=\sup _{z \in C_{n}^{1}\left[t_{0}, t_{1}\right]}\left|g^{*}(t, z, \dot{z})\right|, \\
a=N L_{1} \exp \left(2 M\left(t_{1}-t_{0}\right)\right) .
\end{gathered}
$$

The operator $P$ maps $H$ into itself. As easily seen, all the functions $P(z)(t)$ with $z \in H$ are equicontinuous, since they have uniformly bounded derivatives. We shall now find an estimate for the modulus of continuity of the functions $D P(z)(t)$ for $t, s \in\left[t_{0}, t_{1}\right]$ :

$$
\begin{aligned}
|D P(z)(t)-D P(z)(s)| \leqslant & |A(t) P(z)(t)-A(s) P(z)(s)| \\
& +\left|B(t) K^{*}(t) z(t)-B(s) K^{*}(s) z(s)\right| \\
& +\left|B(t) g^{*}(t, z(t), \dot{z}(t))-B(s) g^{*}(s, z(s), \dot{z}(s))\right| \\
& +|f(t, z(t), \dot{z}(t))-f(s, z(s), \dot{z}(s))| .
\end{aligned}
$$

For the first two terms of the right hand side of (18) we may give the upper estimate as $\beta_{0}(|t-s|)$, where $\beta_{0}$ is a nonnegative function such that $\lim _{h \rightarrow 0^{+}} \beta_{0}(h)=0$ and that it can be chosen independent of the choice of the element $z \in H$. Similarly for the last two terms we have the upper estimate as

$$
k_{2}|\dot{z}(t)-\dot{z}(s)|+\beta_{1}(|t-s|) \text { and } k_{1}|\dot{z}(t)-\dot{z}(s)|+\beta_{2}(|t-s|)
$$

respectively. Letting $\beta=\beta_{0}+\beta_{1}+\beta_{2}$ and $k=k_{1}+k_{2}$, then

$$
|D P(z)(t)-D P(z)(s)| \leqslant k|\dot{z}(t)-\dot{z}(s)|+\beta(|t-s|),
$$

and we infer that

$$
w(D P(z), h) \leqslant k w(D z, h)+\beta(h) .
$$

Hence we conclude that, for any set $E \subset H$,

$$
\mu(P E) \leqslant k \mu(E) \text {. }
$$

Thus, by the Darbo fixed point theorem the operator $P$ has at least one fixed point; therefore there exists a function $z^{*} \in C_{n}^{1}\left[t_{0}, t_{1}\right]$ such that

$$
x^{*}(t)=z^{*}(t)=P\left(z^{*}(t)\right)
$$

This $x^{*}(t)$ satisfies the conditions given in (10) and (12).

REMARK. Regarding the solution of (1), it should be noted that if $f$ satisfies the Lipschitz condition with respect to the vector $x$, then the system (1) is well-posed in the sense of classical theory. We comment that the optimal control obtained in the above theorem is not easy to implement and hence further research is required in this direction. 
The equation (14) represents a nonlinear relation between $z(s)$ and $x(s)$ on $\left[t_{0}, t_{1}\right]$. It is sufficient for the existence of the optimal control (10) that at least one fixed point exists for the nonlinear function (14). Here (14) is equivalent to (15) for the existence of fixed points:

$$
\begin{aligned}
x(t)= & F\left(t, t_{0}\right) x_{0}-\int_{t_{0}}^{t} F(t, s) B(s) K^{*}(s) z(s) d s \\
& -\int_{t_{0}}^{t} F(t, s) B(s) g^{*}(s, z, \dot{z}) d s+\int_{t_{0}}^{t} F(t, s) f(s, z, \dot{z}) d s .
\end{aligned}
$$

If the nonlinear function $f(t, x, \dot{x})$ satisfies the condition (5), then from (12), we have [3]

$$
\begin{aligned}
|g(t, x, y)-g(t, x, \bar{y})| & \leqslant \int_{t}^{t_{1}}|X(t, s) K(s)||f(s, x, y)-f(s, x, \bar{y})| d s \\
& \leqslant b_{1}\left(t_{1}-t_{0}\right) k_{1}|y-\bar{y}|,
\end{aligned}
$$

where $X(t, s)$ is the transition matrix corresponding to the matrix

$$
K(t) B(t) R^{-1}(t) B^{\prime}(t)-A^{\prime}(t) \quad \text { and } \quad b_{1}=\max |X(t, s) K(s)| .
$$

Hence it follows that

$$
\begin{aligned}
\left|g^{*}(t, x, y)-g^{*}(t, x, \bar{y})\right| & \leqslant\left|R^{-1}(t) B^{\prime}(t)\right| b_{1} k_{1}\left(t_{1}-t_{0}\right)|y-\bar{y}| \\
& \leqslant b_{1} b_{2} k_{1}\left(t_{1}-t_{0}\right)|y-\bar{y}|,
\end{aligned}
$$

where $b_{2}=\max \left|R^{-1}(t) B^{\prime}(t)\right|$. Let $k_{2}=b_{1} b_{2} k_{1}\left(t_{1}-t_{0}\right) N$ be such that $0 \leqslant k_{2}<$ $1 / 2$. Then

$$
\left|g^{*}(t, x, y)-g^{*}(t, x, \bar{y})\right| \leqslant\left(k_{2} / N\right)|y-\bar{y}| .
$$

The equation (15) can be written as

$$
x(t)=P(z)(t),
$$

where $P$ is a nonlinear operator on $C_{n}^{1}\left[t_{0}, t_{1}\right]$. This operator is continuous, since all the functions involved in the operator are continuous. Let us consider the closed convex subset

$$
H=\left\{z \in C_{n}^{1}\left[t_{0}, t_{1}\right]:\|z\| \leqslant N_{1},\|D z\| \leqslant N_{2}\right\},
$$

where the positive real constants $N_{1}$ and $N_{2}$ are defined by

$$
\begin{gathered}
N_{1}=\left[\exp \left(M\left(t_{1}-t_{0}\right)\right)\left|x_{0}\right|+N L_{2}\left(t_{1}-t_{0}\right) \exp \left(2 M\left(t_{1}-t_{0}\right)\right)\right. \\
\left.+L \exp \left(2 M\left(t_{1}-t_{0}\right)\right)\right] \exp \left(a\left(t_{1}-t_{0}\right)\right), \\
N_{2}=\left(M+N L_{1}\right) N_{1}+N L_{2}+L,
\end{gathered}
$$




\section{Acknowledgement}

The authors wish to thank the referees for their suggestions.

\section{References}

[1] M. Athans and P. L. Falb, Optimal Control (McGraw Hill, New York, 1966).

[2] F. Colonius and D. Hinrichsen, "Optimal Control of Functional Differential Systems", SIAM J. Control Optim. 16 (1978), 861-879.

[3] R. Conti, Linear Differential Equations and Control (Academic Press, London, 1976).

[4] C. Dacka, "On the Controllability of a Class of Nonlinear Systems", IEEE Trans. Automat. Control 25 (1980), 263-266.

[5] D. L. Lukes, “Optimal Regulation of Nonlinear Systems”, SIAM J. Control Optim. 7 (1969), 75-100.

[6] B. J. Sadovskii, “Limit Compact and Condensing Operators”, Russian Math. Surveys 27 (1972), 85-156.

[7] Y. Yamamoto, "Optimal Control of Nonlinear Systems with Quadratic Performance", $J$. Math. Anal. Appl. 64 (1978), 348-353. 